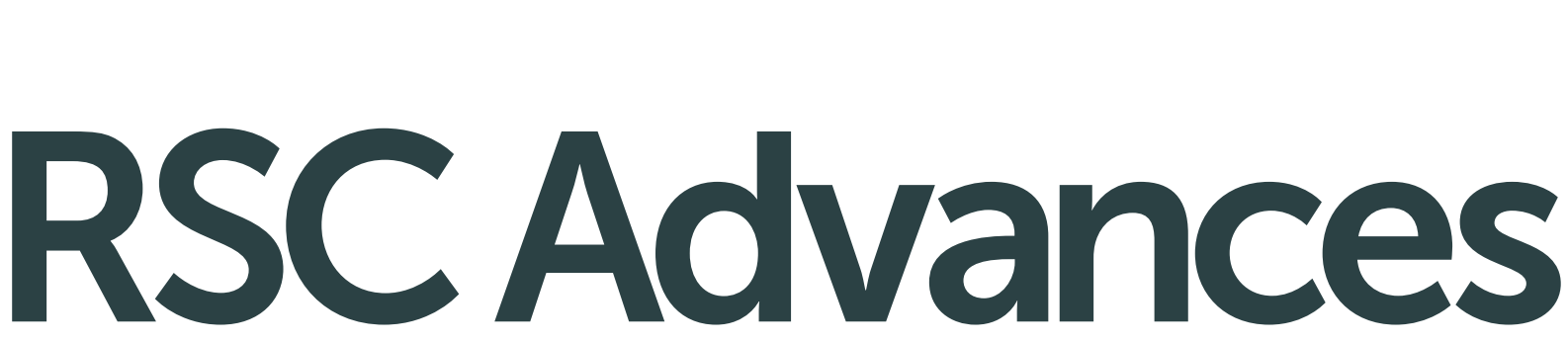

This article can be cited before page numbers have been issued, to do this please use: S. Radic, T. P. Davis, P. C. Ke and F. Ding, RSC Adv., 2015, DOI: 10.1039/C5RA20182A.
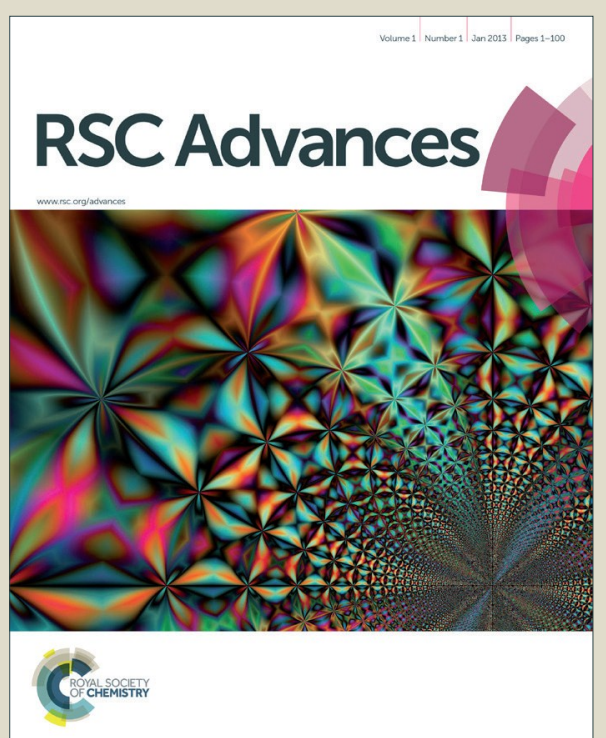

This is an Accepted Manuscript, which has been through the Royal Society of Chemistry peer review process and has been accepted for publication.

Accepted Manuscripts are published online shortly after acceptance, before technical editing, formatting and proof reading. Using this free service, authors can make their results available to the community, in citable form, before we publish the edited article. This Accepted Manuscript will be replaced by the edited, formatted and paginated article as soon as this is available.

You can find more information about Accepted Manuscripts in the Information for Authors.

Please note that technical editing may introduce minor changes to the text and/or graphics, which may alter content. The journal's standard Terms \& Conditions and the Ethical guidelines still apply. In no event shall the Royal Society of Chemistry be held responsible for any errors or omissions in this Accepted Manuscript or any consequences arising from the use of any information it contains. 


\title{
Contrasting effects of nanoparticle-protein attraction on amyloid aggregation
}

\author{
Slaven Radic, ${ }^{1}$ Thomas P Davis, ${ }^{2,3}$ Pu Chun $\mathrm{Ke}^{2}$ and Feng Ding, \\ ${ }^{1}$ Department of Physics and Astronomy, Clemson University, Clemson, SC 29634, USA \\ ${ }^{2}$ ARC Centre of Excellence in Convergent Bio-Nano Science and Technology, Monash Institute of \\ Pharmaceutical Sciences, Monash University, 381 Royal Parade, Parkville, VIC 3052, Australia \\ ${ }^{3}$ Department of Chemistry, Warwick University, Gibbet Hill, Coventry, CV4 7AL, United Kingdom
}

*Correspondence to Feng Ding: fding@clemson.edu

\begin{abstract}
Nanoparticles (NPs) have been experimentally found to either promote or inhibit amyloid aggregation of proteins, but the molecular mechanisms for such complex behaviors remain unknown. Using coarse-grained molecular dynamics simulations, we investigated the effects of varying strength of nonspecific NP-protein attraction on amyloid aggregation of a model protein, the amyloid-beta peptide implicated in Alzheimer's disease. Specifically, with increasing NPpeptide attraction, amyloid aggregation on the NP surface was initially promoted due to increased local protein concentration on the surface and destabilization of the folded state. However, further increase of NP-peptide attraction decreased the stability of amyloid fibrils and reduced their lateral diffusion on NP surface necessary for peptide conformational changes and self-association, thus prohibiting amyloid aggregation. Moreover, we found that the relative concentration between protein and NPs also played an important role on amyloid aggregation. With a high NP/protein ratio, NPs that intrinsically promote protein aggregation may display an inhibitive effect by depleting the proteins in solution while having a low concentration of the proteins on each NP's surface. Our coarse-grained molecular dynamics simulation study offers a molecular mechanism for delineating the contrasting and seemingly conflicting effects of NPprotein attraction on amyloid aggregation and highlights the potential of tailoring antiaggregation nanomedicine against amyloid diseases.
\end{abstract}

\section{Introduction}

With the rapid development of nanotechnology and the increasing ability to engineer nanoparticles (NPs) with diverse functionalities, ${ }^{1}$ much work has been done to exploit the potential use of these novel materials in biomedicine. Applications of nanomedicine range from diagnosis to treatment, impacting drug delivery, ${ }^{2-6}$ imaging, ${ }^{7-10}$ biosensing ${ }^{11}$ and oxidative stress remediation. ${ }^{12}$ Once administrated into biological systems such as the plasma, NPs become surface-coated with sugars, lipids and proteins rich in the media. The molecular complexes with single or multiple layers of biomolecules absorbed onto the NP surface are termed NP-protein corona. ${ }^{13}$ The formation of the protein corona can screen the designed functionality of the NPs ${ }^{14}$ and binding of immunoproteins like opsin may elicit immune responses. ${ }^{15}$ The interactions between NPs and proteins can also induce protein conformational changes to subsequently affect their functions, ${ }^{16,17}$ leading to possible toxic effects. ${ }^{18-22}$ Therefore, it is important to understand NP-protein corona formation and its impact on adsorbed proteins in order to enable the design and vast application of nanomedicine.

Because of the high local concentration of proteins in the corona, NPs have been found to 
promote aberrant aggregation of $\beta-2$ microglobulin, an amyloidogenic protein in serum. ${ }^{23}$ Misfolding and amyloid aggregation of proteins are associated with a wide range of diseases, including Alzheimer's and Huntington's diseases. The amyloid aggregates of different amyloidogenic proteins share the same characteristic cross- $\beta$ structure, where misfolded proteins form extended $\beta$-sheets along the fibril axes. ${ }^{24-29}$ Many recent studies suggest that intermediate oligomers populated along the aggregation pathway rather than the final amyloid fibrils are cytotoxic. ${ }^{30-32}$ Motivated by the possibility of NPs to cross the blood-brain-barrier, ${ }^{33-36}$ much effort has also been devoted to understanding the effect of NPs on protein aggregation in neurodegenerative diseases, such as that of amyloid-beta peptide (A $\beta$ ) in Alzheimer's disease..$^{23,37-45}$ Motivated by advancements in nanotechnology and nanomedicine, many studies have been focused on understanding the impact of NPs with various physicochemical properties on protein aggregation, in order to identify novel NPs that inhibit amyloid aggregation. For instance, drug delivery using NPs with inhibitory effects on amyloid aggregation will have additional therapeutic effects in treating protein misfolding diseases.

Given the diversity of NPs and proteins used in existing experimental studies, both aggregation inhibition and promotion effects have been reported. Amyloid aggregation is a nucleation process, which is characterized by a lag time followed by a sigmoid increase of amyloid fibrils. ${ }^{46}$ It has been showed that various NPs, including copolymer particles, cerium oxide particles, quantum dots, and carbon nanotubes, enhanced fibril formation of $\beta_{2^{-}}$ microglobulin with a shortened aggregation lag time. ${ }^{23}$ Similar effect was seen when $\mathrm{TiO}_{2} \mathrm{NPs}$ were introduced to the $\mathrm{A} \beta$ solution. ${ }^{43}$ It has been suggested that NPs locally increased protein concentration which enhanced the probability of critical aggregation nucleus formation. ${ }^{38-40}$ On the other hand, the fibrillation of $\mathrm{A} \beta$ and islet amyloid peptide (IAPP, a.k.a. amylin) was inhibited by adding polymeric NPs. ${ }^{23,44}$ Single-walled carbon nanotubes were able to prevent the formation of $\beta$-sheets of $A \beta{ }^{47}$ Similarly, negatively charged gold NPs were reported to inhibit $\mathrm{A} \beta$ fibrillization and relieve $\mathrm{A} \beta$ toxicity to neuroblastoma. ${ }^{45}$ Interestingly, it was also reported that, for the same polystyrene NPs, amyloid aggregation of $\mathrm{A} \beta$ was promoted at a high protein to NP ratio but inhibited at lower ratios. ${ }^{37}$ Hence, in order to fully harness the inhibition effects while reducing the aggregation-enhancement effects of NPs, it is crucial to elucidate the driving forces that dictate the drastically different effects of NPs on protein amyloid aggregation (see a summary list of such complex effects in Table 1).

Depending on their core material and surface coating, NPs possess distinct physicochemical properties to dictate their modes of interaction with proteins. The protein aggregation behavior in the presence of NPs is likely determined by inter NP-protein attractions. Using dynamic Monte Carlo (MC) simulations of model peptides (as patchy spherocylinders) interacting with "smooth" surfaces (no atomic structure of the surface), the effects on peptide aggregation kinetics by varying surface attractions were investigated. ${ }^{48}$ A non-linear dependence of aggregation retardation and/or acceleration was found to depend on surface binding affinities and the relative bulk/surface aggregation rate. Because of the highly coarse-grained nature of the model system, detailed molecular insight is still lacking. By modeling the peptide as beads-on-astring in discrete molecular dynamics (DMD) simulations, ${ }^{41}$ the dependence of protein aggregation on protein-protein and protein-NP attraction strengths has been investigated. Therein the NP was modeled as a single bead with nonspecific attraction to the protein atoms. They observed that protein aggregation was enhanced by a stronger protein-NP attraction and the NP surface served as a fibrillation catalyst. However, the experimentally reported inhibition of protein amyloid aggregation was not observed. We postulate that modeling NP as a single bead 
may have omitted some important properties of the NP that may contribute to peptide aggregation on the surface. For instance, the atomic arrangement on the NP surface may limit the lateral diffusion of peptides. In this study, we explicitly modeled the surface atoms of a simplified spherical NP and used A $\beta$ peptide as the model protein. We modeled A $\beta$ peptide by a simplified two-bead-per-residue model, ${ }^{49-51}$ and probed the effects of varying NP-protein attraction strength on $\mathrm{A} \beta$ aggregation. Using DMD simulations, we observed an optimal NPprotein interaction strength with which $\mathrm{A} \beta$ peptides displayed the maximum aggregation on the NP surface. Under the optimal value, increase of NP-protein attraction promoted protein aggregation, while above the optimal value, in contrast, $A \beta$ aggregation was inhibited on the NP surface. In addition, we also examined the concentration dependence of $A \beta$ aggregation on the NP surface, which pointed to a dual effect of NP/protein ratio on protein aggregation. Because the coarse-grained simulations captured the general properties of NP-protein interaction systems, we expect that the obtained mechanistic insight is generally applicable to understand the seemingly conflicting effects of NPs on amyloid aggregation.

\section{Results and Discussion}

In the coarse-grained model for $A \beta$ peptides, each amino acid was represented by the backbone $\mathrm{C}_{\alpha}$ and side-chain $\mathrm{C}_{\beta}$ beads ${ }^{49}$ (Methods). We used a structure-based interaction potential (i.e. the Go model $^{52,53}$ ) to model both intra- and inter-chain interactions of the peptides, where two residues in contact in the native state were assigned an attractive interaction and the same residue pair from different peptides featured the same interaction. Although the effective interaction potentials in the Gō model does not explicitly model physical interactions, e.g. solvation and electrostatics, the model has been found useful to capture important properties of protein aggregation, ${ }^{49-51}$ such as driving forces for aggregation, aggregation pathways, and structural characteristics of amyloid fibrils. We used the NMR structure of A $\beta$ (PDB ID: 1BA4) in the water-micelle environment as the reference structure, where the protein was partially helical (Fig. S1A). For each inter-residue contact between the $\mathrm{C}_{\beta}$ atoms, we assigned an attractive potential with the energy gain of $\varepsilon$. In addition, we also included hydrogen-bond interaction between the backbone $\mathrm{C}_{\alpha}$ beads (Methods). The same two-bead per amino acid model with Gō potential has been used to study amyloid fibril formation ${ }^{54}$ of $\mathrm{A} \beta$ peptides, where the general properties of peptide aggregation were captured. For the NP, we modeled its surface atoms as approximately close-packing on the 2D spherical surface with a diameter of $100 \AA$ (Methods). Although the NP model did not correspond to actual facetted atomic surfaces as observed by electron microscopy, ${ }^{55,56}$ together with the simplified protein model we expected the coarsegrained simulations to capture the essential properties of NP surface upon protein binding without loss of generality. We assigned nonspecific attractive interaction between the NP and the protein atoms with the interaction strength of $\boldsymbol{\varepsilon}_{N \boldsymbol{N}}$. We kept NP atoms static and proteins free to move in the DMD simulations.

Before modeling A $\beta$ aggregation influenced by the NP, we first characterized the folding dynamics of $A \beta$ monomer in the absence of the NP (or in solution) using replica exchange simulations $^{57}$ (Methods). We used the weighted histogram analysis method (WHAM ${ }^{58}$ ) to calculate heat capacity $\left(\mathrm{C}_{\mathrm{v}}\right)$ and radius of gyration $\left(R_{g}\right)$, as functions of temperature in the unit of $\varepsilon / k_{B}$, where $\varepsilon$ is the protein contact energy and $k_{B}$ is the Boltzmann constant (Fig. S1B,C). We observed two peaks in the $\mathrm{C}_{\mathrm{v}}$ plot (Fig. S1B). The first peak at $\mathrm{T} \sim 0.57 \varepsilon / k_{B}$ corresponds to melting of the N-terminal helix (e.g., inset in Fig. 1B). Due to the rigidity of a single helix at low temperatures, unfolding of the N-terminal helix led to decreases of the $R_{g}$ (Fig. S1C). As 
temperature increased further, the rest of the helix started to unfold into a random coil, which in turn resulted in increased $R_{g}$. Therefore, the second peak near $\sim 0.65 \varepsilon / k_{B}$ corresponds to the global unfolding temperature, $T_{m}$. The observed non-corporative folding thermodynamics of A $\beta$ monomer is consistent with previous computational and experimental results. ${ }^{54,59}$ It is wellknown that protein needs to be partially unfolded in order to form $\beta$-sheet rich aggregates. ${ }^{60,61}$ To facilitate observation of protein aggregation in the DMD simulations, we performed our aggregation simulations at $\mathrm{T} \sim 0.655 \varepsilon / k_{B}$ which was slightly above $T_{m}$.

Amyloid aggregation of $\mathbf{A} \beta$ on NP surface with different interaction strengths. We performed aggregation simulations with ten $\mathrm{A} \beta$ peptides in the presence of the model NP by varying the interaction potential between the NP and the peptide atoms $\boldsymbol{\varepsilon}_{N \boldsymbol{P}}$ from $0.1 \varepsilon$ to $0.7 \varepsilon$, with an increment of $0.1 \varepsilon$. For each $\varepsilon_{N P}$, we performed 50 independent DMD simulations with different initial positions and velocities, where ten $A \beta$ monomers were randomly positioned in the vicinity of the spherical NP. To quantify the aggregation process on the NP surface, we monitored the average number of residues per chain that formed inter-chain $\beta$-sheet like structures on the NP surface, $N_{\beta \text {-Res }}$ (Methods). Here, we only included those A $\beta$ peptides that were bound to the NP surface. By averaging over 50 independent runs, we obtained the average $N_{\beta-\text { Res }}$ as a function of the simulation time (Fig. 1A). Usually, protein aggregation in solution is characterized by a lag time phase followed by a rapid elongation phase. ${ }^{62-64}$ Because of the intrinsically disordered nature of the coarse-grained model peptide encoded by the Go potential, the high local concentration of proteins on the NP surface, and also the fact that we simulated at a denaturing temperature (i.e., the nucleation process corresponding to the $\alpha$-to- $\beta$ transition ${ }^{65}$ of individual peptides was negligible), we did not observe the apparent lag time phase in our simulations. The aggregation reached plateau after a rapid elongation phase.

To quantify the aggregation process, we used the sigmoidal function (Methods), routinely used in experimental studies to characterize protein aggregation kinetics, ${ }^{66}$ to fit the computationally derived aggregation kinetics data (dashed lines in Fig. 1A). From the fitting analysis, we obtained the maximal $N_{\beta \text {-Res }}$ as a function of NP-protein interaction strength $\boldsymbol{\varepsilon}_{N P}$ (Fig 1B). We found that the maximal $N_{\beta \text {-Res }}$, which quantifies the amount of $\beta$-sheet aggregation per chain on the NP surface, had a maximum value near $\varepsilon_{N P}=0.3 \varepsilon$. For interactions $\varepsilon_{N P}<0.3 \varepsilon$, the increase of NP-protein attraction resulted in increased protein aggregation. We also observed that with these relatively weak NP-protein interactions the value of $N_{\beta \text {-Res }}$ displayed larger fluctuations compared to the simulations with stronger NP-protein attractions. By computing the number of proteins bound to the NP surface (Fig. S2), we found that large fluctuations resulted from small numbers of proteins on the NP surface, due to weak NP-protein interactions.

When $\boldsymbol{\varepsilon}_{N \boldsymbol{P}}$ is stronger than $0.3 \boldsymbol{\varepsilon}$, the increased interaction between the NP surface atoms and the proteins reduced aggregation (Fig. 1B). Examination of the simulation trajectories indicated that strong NP-protein attractions rendered all $\mathrm{A} \beta$ peptides bound to the NP surface (Fig. S2) but also reduced their mobility. We quantified the mobility of the proteins on the NP surface by computing the protein diffusion coefficient from DMD simulations, $D$ (Fig. S3A). As $\boldsymbol{\varepsilon}_{N P}$ increased, the diffusion coefficient indeed decreased. Interestingly, we observed that the dependence of diffusion coefficient on the NP-peptide interaction strength $\boldsymbol{\varepsilon}_{N \boldsymbol{P}}$ can be described as exponential, $D \sim \exp \left(-n \varepsilon_{N P} / k_{B} T\right)$ (Fig. S3B). Such an exponential dependence is consistent with a hopping mechanism, where the peptide atoms diffuse on the NP surface by hopping between neighboring epitaxy sites, determined by the atomic NP surface (e.g. NP structures in Fig. 1C,D). With reduced diffusion, the proteins were unable to freely diffuse on the NP surface 
and also unable to undergo conformation changes that are important for the formation of interpeptide $\beta$-sheet, thus reducing aggregation (Fig. 1B).

To understand why the peptide aggregation was maximal at $\boldsymbol{\varepsilon}_{N \boldsymbol{P}} \sim 0.3 \boldsymbol{\varepsilon}$, we performed replica exchange DMD simulations of an $\mathrm{A} \beta$ monomer on the NP surface with $\varepsilon_{N P}=0.3 \varepsilon$. Using WHAM, we estimated the specific heat as a function of temperature (Fig. S4A). Compared to A $\beta$ monomer in solution, we noticed a shift of $T_{m}$ (the highest peak) to a lower temperature. The destabilization of the protein on the NP surface was due to stabilization of the unfolded state, where the peptide made more contacts with the NP compared to the folded state. Interestingly, there was a shoulder in the specific heat near temperature $\sim 0.75 \varepsilon / k_{B}$. By calculating the probability of the protein in contact with the NP (Fig. S4B), we found that this shoulder of $C_{v}$ at high temperature corresponded to the dissociation of $\mathrm{A} \beta$ from the NP surface, $T_{d}$. At the intermediate NP-protein interaction strength $\varepsilon_{N P}=0.3 \varepsilon$, the protein was more destabilized on the NP surface compared to that in solution, but still was able to freely diffuse on the NP surface (Fig. S3), thereby promoting the formation of $A \beta$ aggregates (e.g., the snapshot of $A \beta$ aggregates as in Fig. 1C). As the NP-protein attraction increased further, increased destabilization of the protein toward the unfolded states is expected by maximizing contacts between the peptides and the NP. However, such a strong NP-protein attraction also destabilized the amyloid fibril since the peptides tended to interact with the NP rather than among themselves (e.g., snapshot structures from simulations with $\varepsilon_{N P}=0.7 \varepsilon$ in Fig. 2D). Hence, in addition to reduced lateral diffusion, strong NP-protein attraction also enthalpically inhibited the formation of amyloid aggregation.

Taken together, due to the competitions between aggregation-promoting (e.g., increased protein local concentration and destabilization of protein native states) and aggregationinhibiting factors (e.g., deceased diffusion and destabilization of amyloid fibrils), we observed the crossover with the inter-atomic interaction energy between the NPs and the proteins, $\boldsymbol{\varepsilon}_{N P} \sim$ $0.3 \varepsilon$. Various proteins have distinct stabilities that not only depend on their primary, secondary and tertiary structures but also solution conditions, such as temperature, $\mathrm{pH}$ value and salt concentration. The destabilization of protein native states and also amyloid fibrils upon NP binding depends on NP size, shape, and surface curvature. The lateral diffusion of proteins on a NP surface also depends on the physiochemical properties of the surface, such as NP chemical composition, surface atomic arrangement, and functionalization. As a result, we expect that the exact crossover value of inter NP-protein attractions (i.e., binding affinities) may depend on the properties of the protein, the NP, and the solvent of interest.

The dependence of protein aggregation on NP-protein interaction obtained from our coarse-grained simulations with simplified NP and protein models can help explain the contrasting effects of NP-protein attraction on amyloid aggregation reported in the literature (Table 1). For example, $\mathrm{Wu}$ et al. found that $\mathrm{TiO}_{2}$ had a higher affinity to bind $\mathrm{A} \beta$ than other metal-oxides such as $\mathrm{SiO}_{2}, \mathrm{ZrO}_{2}$, and $\mathrm{CeO}_{2} ;$ and the authors observed that $\mathrm{TiO}_{2} \mathrm{NPs}^{43}$ displayed a stronger promoting effect on $\mathrm{A} \beta$ aggregation than the other types of metal-oxide NPs. We believe that these metal-oxide NPs elicit relatively weak NP-protein interactions due to their polar surfaces. Therefore, stronger binding of metal-oxide NPs with A $\beta$ peptides can give rise to increased aggregation. On the other hand, NPs such as graphene, graphene oxide nanosheets, carbon nanotubes and NiPAM:BAM co-polymeric NPs feature strong binding to A $\beta$ peptides and thus strongly hindered amyloid aggregation of $A \beta .{ }^{42,67,68}$ For example, in a recent study combining all-atom molecular dynamics and docking simulations with experimental 
characterizations, ${ }^{69}$ the amyloid fibril formation of $\mathrm{A} \beta$ was found to be disrupted by a strong binding between peptides and graphene/graphene oxide nanosheets enhanced by $\pi-\pi$ stacking between aromatic residues and the carbon-based NP surface. It has also been demonstrated that graphene oxide NPs strongly inhibited the formation of $A \beta$ amyloid fibrils, ${ }^{67}$ where the retardation/inhibition of amyloid fibril formation was revealed by both ThT fluorescence and AFM imaging. By adjusting the composition of NiPAM/BAM co-polymer NPs, Cabaleiro-Lago et al. showed that more hydrophobic 85:15 NiPAM/BAM NPs had a stronger binding to A $\beta$ peptides than the less hydrophobic 50:50 NiPAM/BAM NPs, thereby exerting a greater effect on hindering $\mathrm{A} \beta$ aggregation than the latter.

The concentration-dependence of $\mathbf{A} \boldsymbol{\beta}$ aggregation on NP surface. With relatively weak NPprotein interactions, our simulations suggested that elevated aggregation with increasing $\boldsymbol{\varepsilon}_{N \boldsymbol{P}}$ was due to increased protein concentration on the NP surface (Fig. S2). Next, we evaluated the concentration-dependence of A $\beta$ aggregation on the NP surface with the same interaction strength, $\boldsymbol{\varepsilon}_{N P}=0.3 \varepsilon$. We performed aggregation simulations of various numbers of proteins, from 2 to 10 monomers on the NP surface. At each concentration, we performed 50 independent DMD simulations and computed the average $N_{\beta \text {-Res }}$ as a function of time (Fig. 2A). As the protein concentration increased, we found that the formation of aggregation became faster. For instance, for low peptide concentrations, e.g. the two and four $\mathrm{A} \beta$ simulations, the aggregation was not fully saturated during the course of simulations. Fitted with the sigmoidal function, we obtained the elongation rates, $K_{e}$, as a function of peptide numbers (Fig. 2B). Our results suggested that overall the elongation rate $K_{e}$ increased with increased protein concentration. Interestingly, there was a transition of $K_{e}$ between four and six monomers on the NP surface, after which the concentration dependence of the elongation rate became significantly weaker. This observed transition between slow and fast elongation rate is possibly due to the formation of aggregation nucleus, i.e., oligomers corresponding to the free energy barrier along the aggregation pathway from monomers toward final fibrils. Our simulation results support an aggregation nucleus with the size of approximately four to six $A \beta$ monomers, which is consistent with previous experimental observations of $\mathrm{A} \beta$ aggregation ${ }^{64,70}$.

Competition of A $\beta$ aggregation on NP surface and in solution. Interactions between NPs and peptides may affect the partition of protein concentrations in solution and on the NP surface, and subsequently impact aggregation of proteins on the NP surface and in solution. We performed aggregation simulations with $6 \mathrm{~A} \beta$ monomers in the presence of the NP, where the attractive interactions between the NP and the peptide atoms can be switched on or turned off. A total of 50 independent simulations were carried out starting with each monomer randomly positioned away from the NP surface. We first simulated the system without attractive interaction between the NP and the peptide atoms (only hard-core interaction), and then compared the results with simulation of a weak NP-protein attraction, $\varepsilon_{N P}=0.225 \varepsilon$. In each simulation, we monitored the $N_{\beta-R e s}$ for proteins that were not in contact with the NP, i.e., the aggregation of protein in solution. In the case where the NP atoms had only hard-core interactions with the peptides, we used the same interaction range of $7.5 \AA$ to define the NP-protein contacts. In the aggregation simulations with and without NP-protein attraction, we observed that the formation of inter-peptide hydrogen bonds was highly stochastic (Fig. S5). Although each trajectory followed a sigmoidal-like kinetics of inter-peptide hydrogen bond formation, their lag times varied drastically. For each set of simulations with and without NP-protein attraction, we fitted each of the fifty simulations with the sigmoidal function and computed the corresponding histogram of lag time (Fig. 3A). A 
Kolmogorov-Smirnov test of these two histograms gave a P-value of 0.067 for a null hypothesis of the same underlying distribution, suggesting that the two distributions are different from each other with a confidence level of $\sim 93 \%$. We observed that without NP-protein attraction the distribution of the aggregation lag times in solution was narrower and the corresponding average value was shorter compared to the simulations with a weak attraction.

Since protein aggregation is concentration dependent, we computed the number of proteins in contact with NPs as a function of time by averaging over independent simulations (Fig. 3B). Indeed, the NP-protein attraction increased the local concentration of proteins on the NP surface. As a result, the protein concentration in solution was reduced, which in turn delayed the nucleation of amyloid fibrils in solution. We also computed the formation of $N_{\beta \text {-Res }}$ for proteins on the NP surface as a function of time (Fig. 3C). Upon binding the NP surface due to NP-protein attraction (Fig. 3B), significant protein aggregation was observed in the vicinity of the NP surface compared to simulations without NP-protein attraction. As observed in the previous simulation (Fig. 2A), we did not observe a major lag time of protein aggregation on NP surface at the relatively weak NP-protein attraction strength (Fig. 1). The aggregation of proteins on the NP surface was determined by the amount of proteins diffused to the NP surface.

Complex behavior of protein aggregation in the presence of NPs. Because of the partitioning of proteins in solution and on NP surface induced by NP-protein attraction and also because of the concentration dependence of protein aggregation, our study reveals a mechanistic insight about the complex effects of NPs on protein aggregation. The attraction between NP and protein atoms increased the local concentration of proteins on the NP surface while reducing the protein concentration in solution. When the attraction between the NP and the protein atoms were strong (i.e., right side of the peak in Fig. 1B), amyloid aggregation of the absorbed proteins was inhibited. Together with the reduced amyloid aggregation in solution due to depleted proteins, such NPs always displayed an inhibitive effect on protein aggregation independent of the relative $\mathrm{NP} /$ protein ratio. When the attraction was relatively weak, proteins on the NP surface formed amyloid aggregations in a concentration-dependent manner (Fig. 2). At a high protein/NP ratio with a small amount of NPs introduced to the protein solution, the accumulation of proteins absorbed onto the NP surface could promote protein aggregation (Fig. 4A). With increased NP concentration, the relative protein/NP ratio decreased. As a result, both concentrations of the proteins in solution and on the NP surface decreased compared to the high protein/NP ratio case, which in turn reduced protein aggregations both in solution and on the NP surface (Fig. 4B). Thus, our computational study suggests that NPs with relatively weak attraction to proteins can either promote or hinder protein aggregation depending on the relative concentrations between the protein and the NPs as observed previously in experiments ${ }^{37}$ and in dynamic MC simulations of patchy spherocylinders as model peptides. ${ }^{48}$ Our results are in agreement with a recent experimental work, ${ }^{71}$ where the aggregation of $\alpha$-synuclein in the presence of small unilamellar vesicles (SUV) was studied at different salt concentrations and also different SUV-to-protein concentration ratios. With increased salt concentrations, the dissociation constant of proteins from the SUV surface increased from 0.35 to $11 \mu \mathrm{M}$, indicated a decreased binding between SUVs and $\alpha$-synuclein. Such a decreased protein-SUV binding led to decreased rate of amyloid fibril formation. More importantly, an enhancement in aggregation was observed when the relative SUV-to-protein concentration ratio was low in the range from 2 to 15, but under higher SUV-to-protein concentration ratios (from 15 to 40) the rate of fibril formation decreased.

Given the differences between our DMD simulations and previous MC studies ${ }^{48}$ in terms 
of peptide and NP surface models as well as NP-peptide attraction scales, it is not straightforward to directly compare the results from the two computational studies. Based on the relative aggregation kinetics on NP surface (Fig. 1,2) and in solution (Fig. 3), we note that our results of $\varepsilon_{N P}$ at $0.1 \sim 0.3 \varepsilon$ might correspond to the previous MC simulations with attractions or high attractions between model peptides and surface, where the aggregation is promoted/accelerated at the NP surface compared to aggregation in solution or bulk. The higher resolution of both peptide and NP surface models in the current study allows us to observe the aggregation-inhibition due to strong peptide and protein absorption onto the NP surface (e.g. graphene and graphene oxide), ${ }^{42,67}$ the phenomena of which have not been captured by other computational models.

\section{Conclusion}

We have applied coarse-grained DMD simulations to systematically study the effects of nonspecific NP-peptide attractions on peptide aggregation. Although the simplified NP/protein model may lack the chemical details of specific NPs and peptides, it captures the general properties of NP-protein systems. In addition, the structure-based Gō interaction potentials has been utilized to model the generic properties of peptide aggregation in the presence of NPs instead of predicting specific aggregation pathways of $A \beta$. The corresponding coarse-grained simulations recapitulate the experimentally-observed aggregation promotion and/or inhibition by various NPs and provide the corresponding molecular insights. Our computational studies demonstrated that NP-protein interactions contribute to multiple factors important for amyloid aggregation of proteins on the NP surface. These factors include protein concentration, stability of protein folded structures, stability of amyloid fibrils, and protein mobility on the NP surface. Increases in NP-protein attraction led to increased surface protein concentration, destabilization of protein folded states, destabilization of amyloid fibrils, and reduction of protein lateral diffusion. While increase of protein concentration and destabilization of protein folded states promoted amyloid aggregation, destabilization of amyloid fibrils and reduction of lateral diffusion inhibited protein aggregation. Consequential to the interplay between these competing factors, we observed an initial aggregation promotion followed by aggregation inhibition depending on the interaction strength between the NP and the protein atoms. Additionally, our study indicated that the relative concentrations between proteins and NPs also serve an important role on amyloid aggregation. Under a high NP/protein ratio, NPs that intrinsically promote protein aggregation may display an inhibitive effect by depleting the proteins in solution but effectively having a low protein concentration on the NP surface. Therefore, in order to characterize the intrinsic effects of NPs on amyloid aggregation, it is necessary to study a wide range of NP-protein ratios.

\section{Methods}

Discrete molecular dynamics (DMD). More detailed description of DMD algorithm can be found in literature ${ }^{72,73}$. Shortly, inter-atomistic interactions were modeled by square step potential. Bonds, bond angles and dihedrals were modeled by infinitely deep square-well potential. During a simulation, an atom would have constant velocity until it reached boundaries of the potential, at which instant it changed velocity according to energy, momentum and angular momentum conservation laws. For each step in the simulations, possible encounters of atoms and 
potential wells were sorted so subsequent collision could be predicted. Compared to traditional molecular dynamics simulation (MD) continuous potential functions, DMD potentials are discretized by step functions, reducing DMD simulations to event-driven molecular dynamics simulations. Better sampling efficiency of DMD over MD originates mainly from the rapid processing of collision events and localized updates of collisions (only collided atoms are required to update at each collision). ${ }^{74}$

Two-bead model of the peptide. We modeled $A \beta$ peptide using the two-bead per residue model. ${ }^{49,60}$ Each amino acid was modeled using one bead for $\mathrm{C}_{\alpha}$ (backbone carbon) and another for $\mathrm{C}_{\beta}$ (side chain). Intra-molecular bonds along the peptide were assigned to reflect protein geometry. Side chain-side chain interactions were modeled using the structure-based potential, which favored observation of native state interactions. The interaction strength between native contacts was set to $\varepsilon$, while the attractions between $\mathrm{C}_{\beta}$ atoms were assigned with the hard-core distance of $D_{h c}=3 \AA$ and the interaction range $D_{I R}=7.5 \AA$. Also, hydrogen bonds were included in this model between the backbone atoms ${ }^{60}$. Each $\mathrm{C}_{\alpha}$ could form maximum two hydrogen bonds with another $\mathrm{C}_{\alpha}$ atom, and two bonds formed by one $\mathrm{C}_{\alpha}$ were co-linear in order to model the angular dependence of hydrogen bonds. Other inter-atomic interactions were modeled by simple hard-core collision with the hard-core distance of $3 \AA$.

In our coarse-grained DMD simulations, the unit of length was angstrom $\left(10^{-10}\right.$ meter $)$. Assuming the energy unit $\varepsilon \sim 1 \mathrm{kcal} / \mathrm{mol}$ (i.e., the energy gain for each Gō contact) and mass unit $\sim 50$ Delton (the average mass of the coarse-grained $\mathrm{C}_{\alpha}$ and $\mathrm{C}_{\beta}$ beads), the time unit (t.u.) was determined as approximately 353 femtoseconds.

NP model. The NP was modeled as two layers close-packed all-atom sphere of $D=100 \AA$ in diameter. The VDW radius of the atom was considered to be $r=1.8 \AA$. To determine this number we used formula $N=\left(D^{2}+(D-2 r \sqrt{3 / 2})^{2}\right) \rho_{2 d} / r^{2}$, where $\rho_{2 \mathrm{~d}}$ denotes the density of twodimensional packing, 0.9069 . The number of atoms calculated needed to form 2-layered closed packing was $N \sim 5217$. Initially, the atoms were uniformly distributed around the center in the $\theta$ and $\varphi$ coordinates, while distribution along the radial direction was Gaussian with the center of $50 \AA$ and standard deviation of $7 \AA$. All atoms were assigned hard-core collision interaction with each other with $D_{\mathrm{hc}}=3.6 \AA$, and were confined to the surface of the NP with a potential step between $46.88 \AA$ and $50 \AA$ to the core bead, which was kept static in DMD simulations. Then, relaxation simulations were performed at $\mathrm{T}=0.6 \varepsilon / k_{B}$ for 2 million time units, $0.7 \varepsilon / k_{B}$ for next 1 million time units, and at $\mathrm{T}=0.85 \varepsilon / k_{B}$ for the last 1 million time units. Finally, all atoms were approximately packed closely into two layers (Fig. S6). We assigned attractive interactions between any NP atoms and peptide atoms with the interaction range $\mathrm{D}_{\mathrm{IR}}=7.5 \AA$, and the hardcore distance of $\mathrm{D}_{\mathrm{hc}}=3.6 \AA$.

Simulations of $\mathbf{A} \boldsymbol{\beta}$ monomer. We used a $200 \times 200 \times 200 \AA^{3}$ simulation box with periodic boundary conditions. The molecular system was initially equilibrated at $\mathrm{T}=0.45 \varepsilon / k_{B}$ for $10^{5}$ time units. Eight replica with temperatures $\mathrm{T}=0.45,0.50,0.55,0.60,0.65,0.70,0.75,0.80$, and $0.85 \varepsilon / k_{B}$ for $3 \times 10^{6}$ time units.

Simulations of A $\boldsymbol{\beta}$ aggregation on NP surface. We used a simulation box of $205 \times 205 \times 205 \AA^{3}$ with periodic boundary conditions. The NP was placed at the center. A $\beta$ peptides were randomly placed in proximity of the NP surface. Prior to production simulations, we equilibrated the 
system at $\mathrm{T}=0.65 \varepsilon / k_{B}$ for $10^{5}$ time units. For each of interaction strengths $-0.1 \varepsilon,-0.2 \varepsilon,-0.3 \varepsilon,-$ $0.4 \varepsilon,-0.5 \varepsilon,-0.6 \varepsilon$ and $-0.7 \varepsilon$, we performed 50 independent simulations with different initial conditions for the duration of $1 \times 10^{6}$ time units.

Calculation of residues forming $\boldsymbol{\beta}$-sheets. In order to measure the extent of amyloid aggregation, we counted the number of residues that were aligned to satisfy a $\beta$-sheet-like (either parallel or anti-parallel) geometry. We considered two residues $(i, j)$ for different chains in $\beta$ contact if the distance between their corresponding $C_{\beta}$ atoms was less than $7.5 \AA$, and their corresponding nearest neighbors $(i+1, j+1)$ and $(i-1, j-1)$ were in contact for parallel-like $\beta$-sheet, or $(i+1, j-1)$ and $(i-1, j+1)$ were in contact for anti-parallel-like $\beta$-sheet.

A protein was counted as on the NP surface if any of the protein atoms made contact with the NP surface atoms with a cutoff distance of $7.5 \AA$. We used the empirical sigmoidal function to fit the aggregation kinetics data: $y=(A-B) /\left(1+\exp \left(-k\left(t-t_{0}\right)\right)\right)+B$, using OriginLab 9.1 (www.originlab.com). Here, fitting parameter A corresponded to the maximum aggregate. The lag time $t_{\text {lag }}$ was defined as $t_{\text {lag }}=\mathrm{t}_{0}-2 / k$. In cases where there was no obvious lag time for aggregation on the NP surface, we set $t_{0}$ as zero in the fitter (e.g. Figs. 1,2).

\section{Acknowledgment}

The work was supported in part by NSF CBET-1232724, NIH R15ES022766-01A1, and Clemson University startup funds. All simulations were performed on the Palmetto high performance cluster, which is managed and maintained by Clemson University CCIT.

\section{References}

1 M. J. Pitkethly, Mater. Today, 2004, 7, 20-29.

2 W. H. De Jong and P. J. Borm, Int. J. Nanomedicine, 2008, 3, 133-149.

3 S. Goenka, V. Sant and S. Sant, J. Controlled Release, 2014, 173, 75-88.

4 J. A. Hubbell and A. Chilkoti, Science, 2012, 337, 303-305.

5 D. M. Webster, P. Sundaram and M. E. Byrne, Eur. J. Pharm. Biopharm., 2013, 84, 1-20.

6 G. Zhang, X. Zeng and P. Li, J. Biomed. Nanotechnol., 2013, 9, 741-750.

7 X. Li, R. Wang, F. Zhang, L. Zhou, D. Shen, C. Yao and D. Zhao, Sci. Rep., 2013, 3.

8 R. Liang, M. Wei, D. G. Evans and X. Duan, Chem. Commun., 2014, 50, 14071-14081.

9 P. Sharma, S. Brown, G. Walter, S. Santra and B. Moudgil, Adv. Colloid Interface Sci., 2006, 123-126, 471-485.

10F. Peng, Y. Su, Y. Zhong, C. Fan, S.-T. Lee and Y. He, Acc. Chem. Res., 2014, 47, 612-623.

11 M. Holzinger, A. Le Goff and S. Cosnier, Front. Chem., 2014, 2.

12B. Srdjenovic, V. Milic-Torres, N. Grujic, K. Stankov, A. Djordjevic and V. Vasovic, Toxicol. Mech. Methods, 2010, 20, 298-305.

13 T. Cedervall, I. Lynch, S. Lindman, T. Berggård, E. Thulin, H. Nilsson, K. A. Dawson and S. Linse, Proc. Natl. Acad. Sci. U. S. A., 2007, 104, 2050-2055.

14 M. P. Monopoli, D. Walczyk, A. Campbell, G. Elia, I. Lynch, F. Baldelli Bombelli and K. A. Dawson, J. Am. Chem. Soc., 2011, 133, 2525-2534.

15X. Cai, S. Conley and M. Naash, Vision Res., 2008, 48, 319-324.

16M. Lundqvist, J. Stigler, G. Elia, I. Lynch, T. Cedervall and K. A. Dawson, Proc. Natl. Acad.

Sci. U. S. A., 2008, 105, 14265-14270. 
17E. Hellstrand, I. Lynch, A. Andersson, T. Drakenberg, B. Dahlbäck, K. A. Dawson, S. Linse and T. Cedervall, FEBS J., 2009, 276, 3372-3381.

18L. Q. Chen, L. Fang, J. Ling, C. Z. Ding, B. Kang and C. Z. Huang, Chem. Res. Toxicol., 2015.

19J. C. Bonner, R. M. Silva, A. J. Taylor, J. M. Brown, S. C. Hilderbrand, V. Castranova, D. Porter, A. Elder, G. Oberdörster, J. R. Harkema, L. A. Bramble, T. J. Kavanagh, D. Botta, A. Nel and K. E. Pinkerton, Environ. Health Perspect., 2013, 121, 676-682.

20R. Chen, S. Radic, P. Choudhary, K. G. Ledwell, G. Huang, J. M. Brown and P. Chun Ke, Appl. Phys. Lett., 2012, 101, 133702.

21 T. Hirai, Y. Yoshioka, K. Ichihashi, T. Mori, N. Nishijima, T. Handa, H. Takahashi, S.

Tsunoda, K. Higashisaka and Y. Tsutsumi, J. Immunol., 2014, 192, 118.19-118.19.

22 Y. K. Lee, E.-J. Choi, T. J. Webster, S.-H. Kim and D. Khang, Int. J. Nanomedicine, 2015, 10, 97-113.

23 S. Linse, C. Cabaleiro-Lago, W.-F. Xue, I. Lynch, S. Lindman, E. Thulin, S. E. Radford and

K. A. Dawson, Proc. Natl. Acad. Sci., 2007, 104, 8691-8696.

24R. Nelson, M. R. Sawaya, M. Balbirnie, A. Ø. Madsen, C. Riekel, R. Grothe and D.

Eisenberg, Nature, 2005, 435, 773-778.

25 J. P. Cleary, D. M. Walsh, J. J. Hofmeister, G. M. Shankar, M. A. Kuskowski, D. J. Selkoe and K. H. Ashe, Nat. Neurosci., 2005, 8, 79-84.

26I. Benilova, E. Karran and B. De Strooper, Nat. Neurosci., 2012, 15, 349-357.

27 L. V. Kalia, S. K. Kalia, P. J. McLean, A. M. Lozano and A. E. Lang, Ann. Neurol., 2013, 73, 155-169.

28 G. Hoffner, S. Souès and P. Djian, Prion, 2007, 1, 26-31.

29L. G. Nucifora, K. A. Burke, X. Feng, N. Arbez, S. Zhu, J. Miller, G. Yang, T. Ratovitski, M. Delannoy, P. J. Muchowski, S. Finkbeiner, J. Legleiter, C. A. Ross and M. A. Poirier, J. Biol. Chem., 2012, 287, 16017-16028.

30M. Stefani, FEBS J., 2010, 277, 4602-4613.

31 B. Caughey and P. T. Lansbury, Annu. Rev. Neurosci., 2003, 26, 267-298.

32 J. Moreth, K. S. Kroker, D. Schwanzar, C. Schnack, C. A. F. von Arnim, B. Hengerer, H. Rosenbrock and L. Kussmaul, Biochemistry (Mosc.), 2013, 52, 1466-1476.

33 Y. Cheng, Q. Dai, R. A. Morshed, X. Fan, M. L. Wegscheid, D. A. Wainwright, Y. Han, L. Zhang, B. Auffinger, A. L. Tobias, E. Rincón, B. Thaci, A. U. Ahmed, P. C. Warnke, C. He and M. S. Lesniak, Small Weinh. Bergstr. Ger., 2014, 10, 5137-5150.

34 J. Li, P. Cai, A. Shalviri, J. T. Henderson, C. He, W. D. Foltz, P. Prasad, P. M. Brodersen, Y. Chen, R. DaCosta, A. M. Rauth and X. Y. Wu, ACS Nano, 2014, 8, 9925-9940.

35 J. Tang, L. Xiong, G. Zhou, S. Wang, J. Wang, L. Liu, J. Li, F. Yuan, S. Lu, Z. Wan, L. Chou and T. Xi, J. Nanosci. Nanotechnol., 2010, 10, 6313-6317.

36C.-M. Lin, Recent Pat. Nanotechnol., 2012, 6, 105-113.

37 C. Cabaleiro-Lago, F. Quinlan-Pluck, I. Lynch, K. A. Dawson and S. Linse, ACS Chem.

Neurosci., 2010, 1, 279-287.

38 O. S. C Cabaleiro-Lago, Langmuir ACS J. Surf. Colloids, 2011, 28, 1852-7.

39 M. Zaman, E. Ahmad, A. Qadeer, G. Rabbani and R. H. Khan, Int. J. Nanomedicine, 2014, 9, 899-912.

40 V. L. Colvin and K. M. Kulinowski, Proc. Natl. Acad. Sci., 2007, 104, 8679-8680.

41 S. Auer, A. Trovato and M. Vendruscolo, PLoS Comput Biol, 2009, 5, e1000458.

42 H. Li, Y. Luo, P. Derreumaux and G. Wei, Biophys. J., 2011, 101, 2267-2276. 
43 W. Wu, X. Sun, Y. Yu, J. Hu, L. Zhao, Q. Liu, Y. Zhao and Y. Li, Biochem. Biophys. Res.

Commun., 2008, 373, 315-318.

44 C. Cabaleiro-Lago, I. Lynch, K. A. Dawson and S. Linse, Langmuir, 2010, 26, 3453-3461. 45 Y.-H. Liao, Y.-J. Chang, Y. Yoshiike, Y.-C. Chang and Y.-R. Chen, Small Weinh. Bergstr.

Ger., 2012, 8, 3631-3639.

46P. Hortschansky, V. Schroeckh, T. Christopeit, G. Zandomeneghi and M. Fändrich, Protein

Sci. Publ. Protein Soc., 2005, 14, 1753-1759.

47 J. Guo, J. Li, Y. Zhang, X. Jin, H. Liu and X. Yao, PLoS ONE, 2013, 8.

48R. Vácha, S. Linse and M. Lund, J. Am. Chem. Soc., 2014, 136, 11776-11782.

49F. Ding, N. V. Dokholyan, S. V. Buldyrev, H. E. Stanley and E. I. Shakhnovich, Biophys. J., $2002,83,3525-3532$.

50 S. Yang, S. S. Cho, Y. Levy, M. S. Cheung, H. Levine, P. G. Wolynes and J. N. Onuchic,

Proc. Natl. Acad. Sci. U. S. A., 2004, 101, 13786-13791.

51 P. I. Zhuravlev, G. Reddy, J. E. Straub and D. Thirumalai, J. Mol. Biol., 2014, 426, 2653-

2666.

52 Y. Ueda, H. Taketomi and N. Gō, Biopolymers, 1978, 17, 1531-1548.

53 H. Taketomi, Y. Ueda and N. Gō, Int. J. Pept. Protein Res., 1975, 7, 445-459.

54 S. Peng, F. Ding, B. Urbanc, S. V. Buldyrev, L. Cruz, H. E. Stanley and N. V. Dokholyan,

Phys. Rev. E Stat. Nonlin. Soft Matter Phys., 2004, 69, 041908.

55 N. Tian, Z.-Y. Zhou, S.-G. Sun, Y. Ding and Z. L. Wang, Science, 2007, 316, 732-735.

56 J. R. Morones, J. L. Elechiguerra, A. Camacho, K. Holt, J. B. Kouri, J. T. Ramírez and M. J.

Yacaman, Nanotechnology, 2005, 16, 2346-2353.

57 Y. Sugita and Y. Okamoto, Chem. Phys. Lett., 1999, 314, 141-151.

58 S. Kumar, J. M. Rosenberg, D. Bouzida, R. H. Swendsen and P. A. Kollman, J. Comput.

Chem., 1992, 13, 1011-1021.

59D. B. Teplow, N. D. Lazo, G. Bitan, S. Bernstein, T. Wyttenbach, M. T. Bowers, A.

Baumketner, J.-E. Shea, B. Urbanc, L. Cruz, J. Borreguero and H. E. Stanley, Acc. Chem.

Res., 2006, 39, 635-645.

60F. Ding, N. V. Dokholyan, S. V. Buldyrev, H. E. Stanley and E. I. Shakhnovich, J. Mol. Biol., 2002, 324, 851-857.

61 F. Ding, Y. Furukawa, N. Nukina and N. V. Dokholyan, J. Mol. Biol., 2012, 421, 548-560.

62 J. T. Jarrett, E. P. Berger and P. T. Lansbury, Ann. N. Y. Acad. Sci., 1993, 695, 144-148.

63 D. M. Walsh, A. Lomakin, G. B. Benedek, M. M. Condron and D. B. Teplow, J. Biol. Chem., 1997, 272, 22364-22372.

64 A. Lomakin, D. B. Teplow, D. A. Kirschner and G. B. Benedek, Proc. Natl. Acad. Sci. U. S. A., 1997, 94, 7942-7947.

65F. Ding, J. M. Borreguero, S. V. Buldyrey, H. E. Stanley and N. V. Dokholyan, Proteins, 2003, 53, 220-228.

66 J. D. Harper and P. T. Lansbury, Annu. Rev. Biochem., 1997, 66, 385-407.

67 M. Mahmoudi, O. Akhavan, M. Ghavami, F. Rezaee and S. M. A. Ghiasi, Nanoscale, 2012, 4, $7322-7325$.

68C. Cabaleiro-Lago, F. Quinlan-Pluck, I. Lynch, S. Lindman, A. M. Minogue, E. Thulin, D. M.

Walsh, K. A. Dawson and S. Linse, J. Am. Chem. Soc., 2008, 130, 15437-15443.

69Z. Yang, C. Ge, J. Liu, Y. Chong, Z. Gu, C. A. Jimenez-Cruz, Z. Chai and R. Zhou,

Nanoscale, 2015, 7, 18725-18737. 
70 A. Lomakin, D. S. Chung, G. B. Benedek, D. A. Kirschner and D. B. Teplow, Proc. Natl. Acad. Sci. U. S. A., 1996, 93, 1125-1129.

71 C. Galvagnion, A. K. Buell, G. Meisl, T. C. T. Michaels, M. Vendruscolo, T. P. J. Knowles and C. M. Dobson, Nat. Chem. Biol., 2015, 11, 229-234.

72 D. C. Rapaport, The art of molecular dynamics simulation, Cambridge University Press, Cambridge, UK; New York, NY, 2004.

73 M. P. Allen and D. J. Tildesley, Computer Simulation of Liquids, Oxford University Press, Oxford England; New York, Reprint edition., 1989.

74 N. V. Dokholyan, Computational Modeling of Biological Systems: From Molecules to Pathways, Springer Science \& Business Media, 2012.

75 D. Zhang, O. Neumann, H. Wang, V. M. Yuwono, A. Barhoumi, M. Perham, J. D. Hartgerink, P. Wittung-Stafshede and N. J. Halas, Nano Lett., 2009, 9, 666-671.

76 S. Li, L. Wang, C. C. Chusuei, V. M. Suarez, P. L. Blackwelder, M. Micic, J. Orbulescu and R. M. Leblanc, Chem. Mater., 2015, 27, 1764-1771. 


\section{Tables}

Table 1. A list of experimental studies illustrating the complex effects of NPs on protein amyloid aggregation, including promotion, inhibition, and also duel effects.

\begin{tabular}{|c|c|c|}
\hline Nanoparticles & Proteins & Effects on Amyloid Aggregation \\
\hline $\begin{array}{l}\text { Multi-walled CNT, } \\
\text { QDs, Copolymer NP, } \\
\mathrm{CeO}_{2} \mathrm{NP}^{23}\end{array}$ & $\beta-2$ microglobulin & \multirow[t]{3}{*}{ Promotion } \\
\hline $\mathrm{TiO}_{2} \mathrm{NP}^{43}$ & $\mathrm{~A} \beta$ & \\
\hline $\mathrm{AuNP}^{75}$ & lysozyme & \\
\hline Graphene oxide ${ }^{67}$ & $\mathrm{~A} \beta$ & \multirow{5}{*}{ Inhibition } \\
\hline AuNP 45 & $\mathrm{~A} \beta$ & \\
\hline $\mathrm{CNT}^{42}$ & $\mathrm{~A} \beta_{16-22}$ & \\
\hline Carbon Dots $^{76}$ & Insulin & \\
\hline Polymeric NP ${ }^{68}$ & $\mathrm{~A} \beta$ & \\
\hline Polystyrene $\mathrm{NP}^{37}$ & $\mathrm{~A} \beta$ & Either promotion or inhibition \\
\hline
\end{tabular}




\section{Figures}
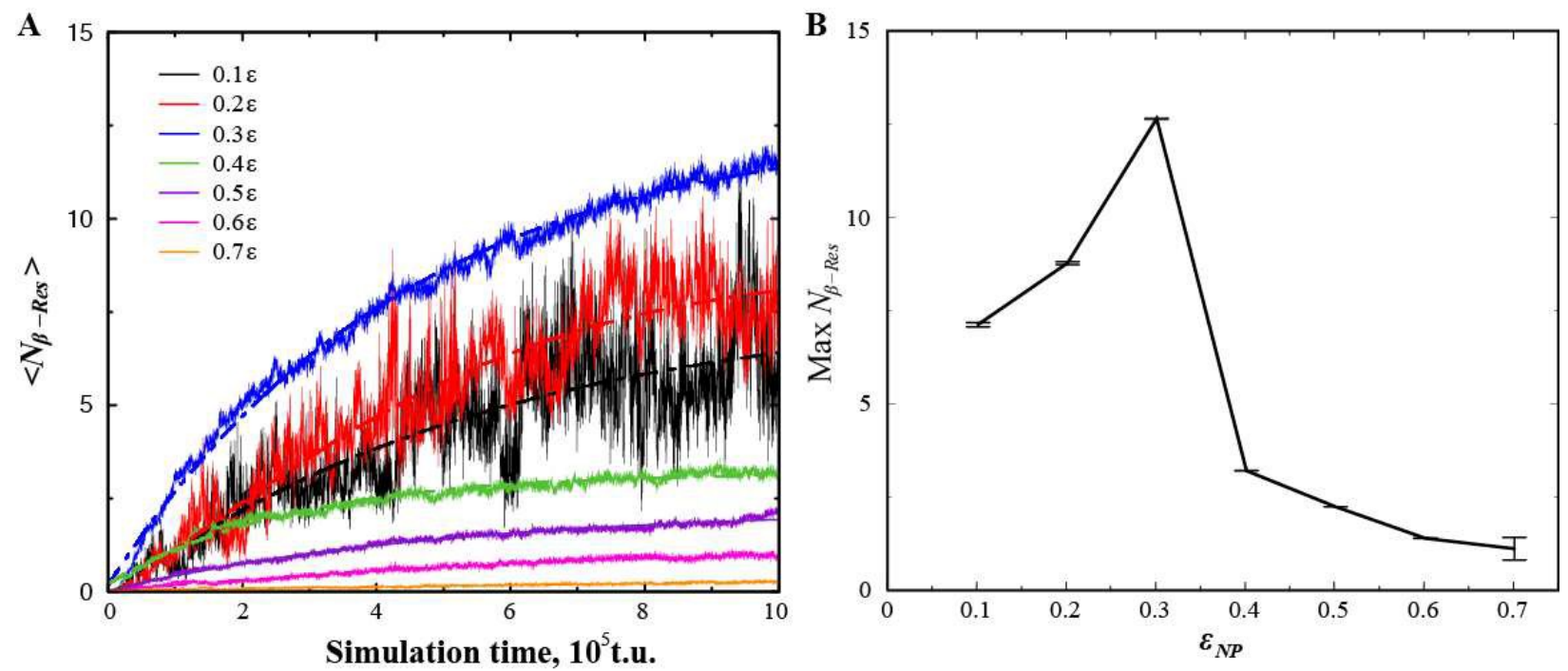

C

\section{D}
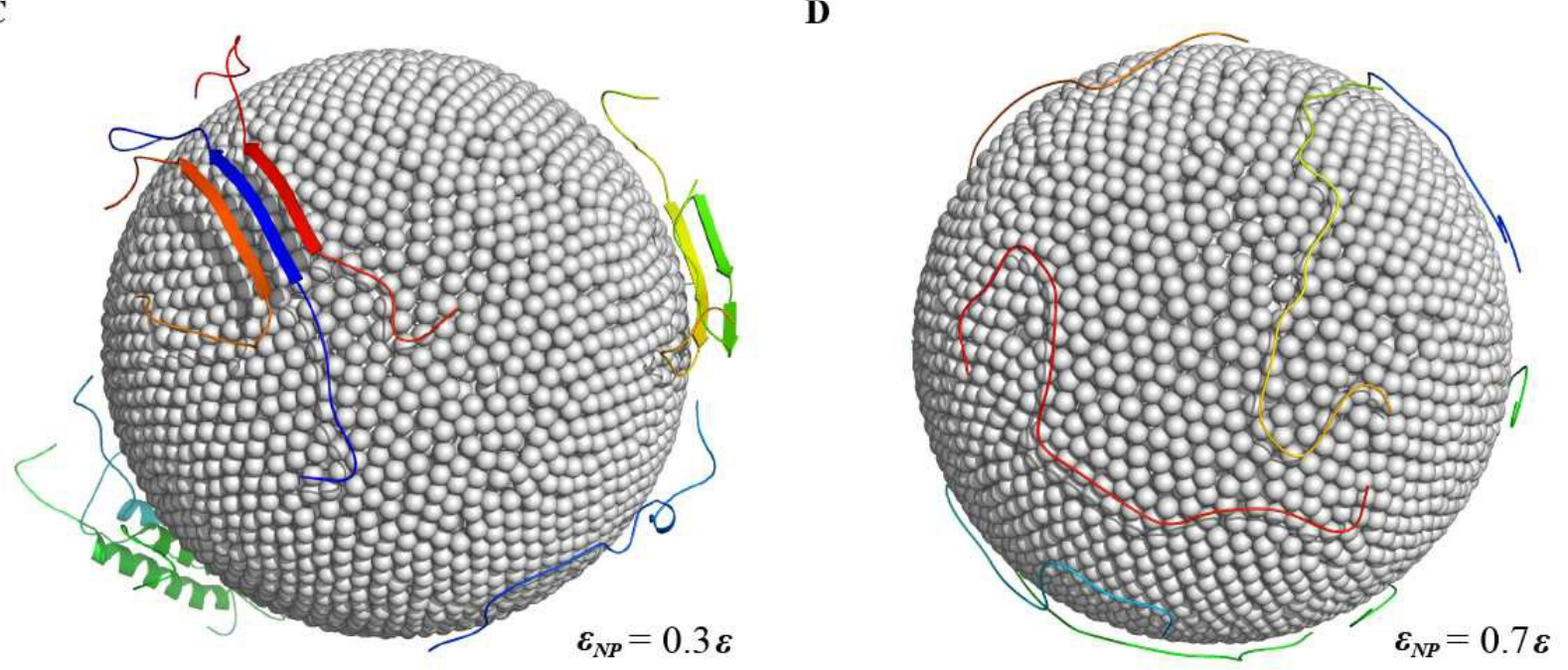

Figure 1. The dependence of $A \beta$ aggregation on NP-protein interaction strength. (A) For simulations of each assigned NP-peptide interaction strength $\varepsilon_{N P}$, the average number of residues per chain that formed inter-peptide beta-sheets, $N_{\beta-R e s}$, was computed as a function of simulation time. All 50 independent simulations were used for the averaging. The fitted sigmoidal curves were shown as dashed lines. (B) The maximum $N_{\beta \text {-Res }}$ as a function of NP-peptide interaction strength $\varepsilon_{N P}$ was estimated from sigmoidal fitting (Fig. S1). Representative snapshot structures from simulations of $\varepsilon_{N P}=0.3 \varepsilon(\mathrm{C})$ and $\varepsilon_{N P}=0.7 \varepsilon(\mathrm{D})$. The surface atoms of the NP are shown as spheres and proteins in cartoon representation. Different peptides are colored differently. 
A

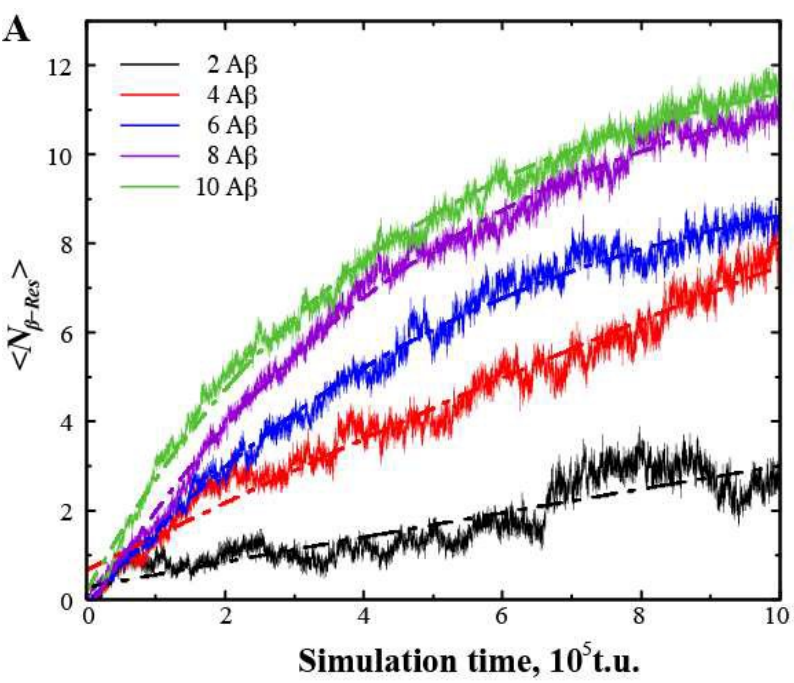

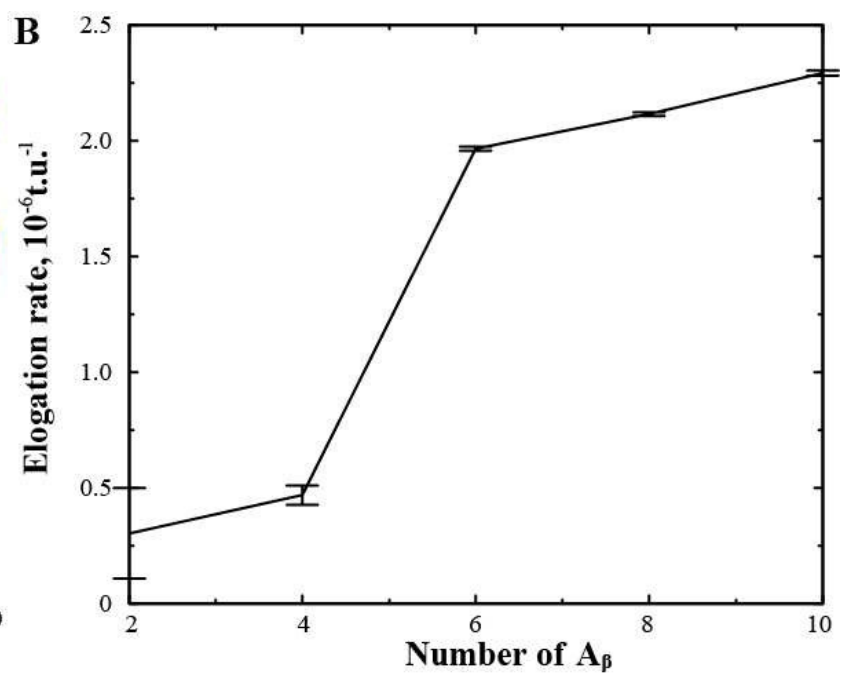

Figure 2. The dependence of $A \beta$ aggregation on peptide concentration on NP surface. (A) The average $\beta$-sheet content per chain, $N_{\beta \text {-Res }}$, as a function of simulation time for simulations with different numbers of peptides adsorbed on the NP surface. The dashed lines correspond to the sigmoidal fitting. (B) The elongation rates derived from curve fitting of (A). 

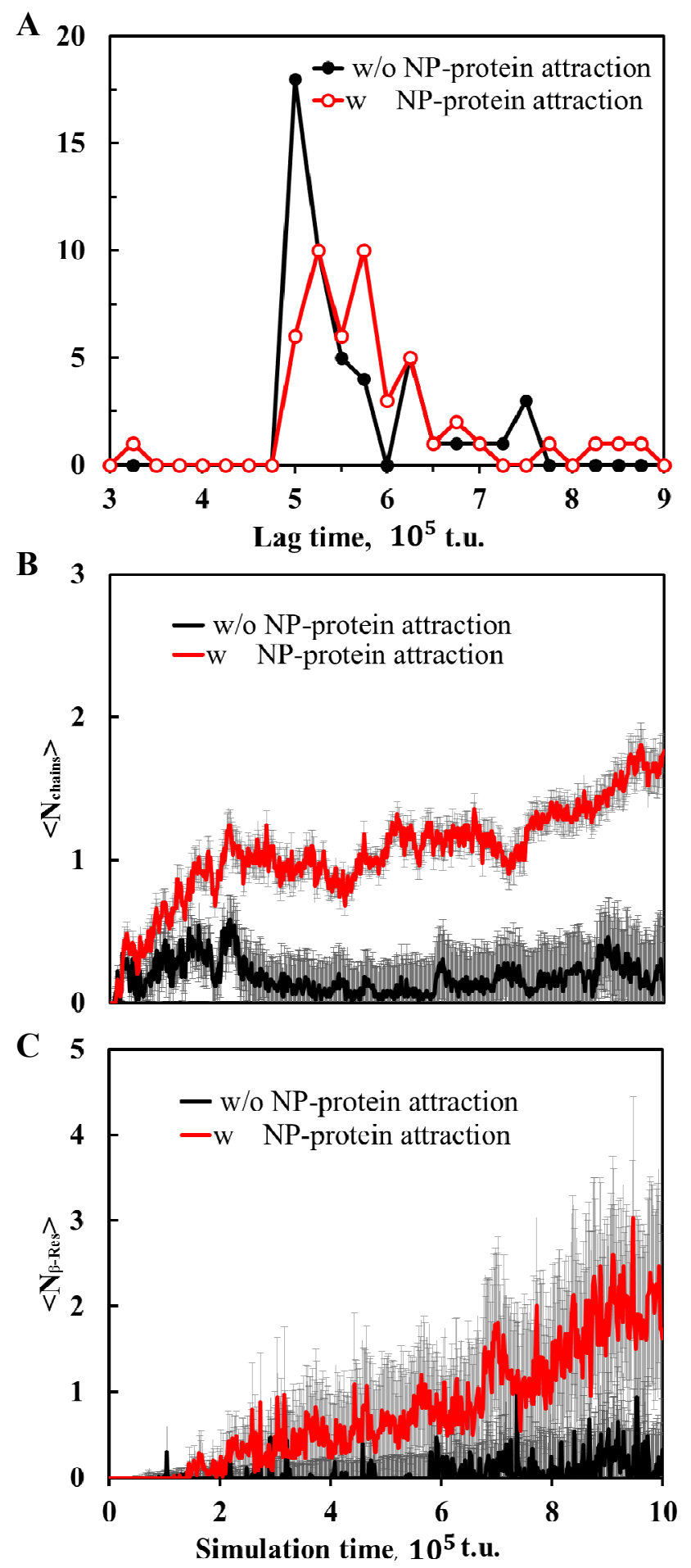

Figure 3. Competition of $A \boldsymbol{\beta}$ aggregation on NP surface and in solution. (A) Histograms of $\mathrm{A} \beta$ aggregation lag times in solution, where the interactions between NP and peptide atoms were switched off and turned on. (B) Average number of chains in the vicinity of the NP surface over time. (C) Aggregation trajectories of amyloid beta in the vicinity of the NP surface. The error bars correspond to standard deviations computed from independent aggregation simulations. 

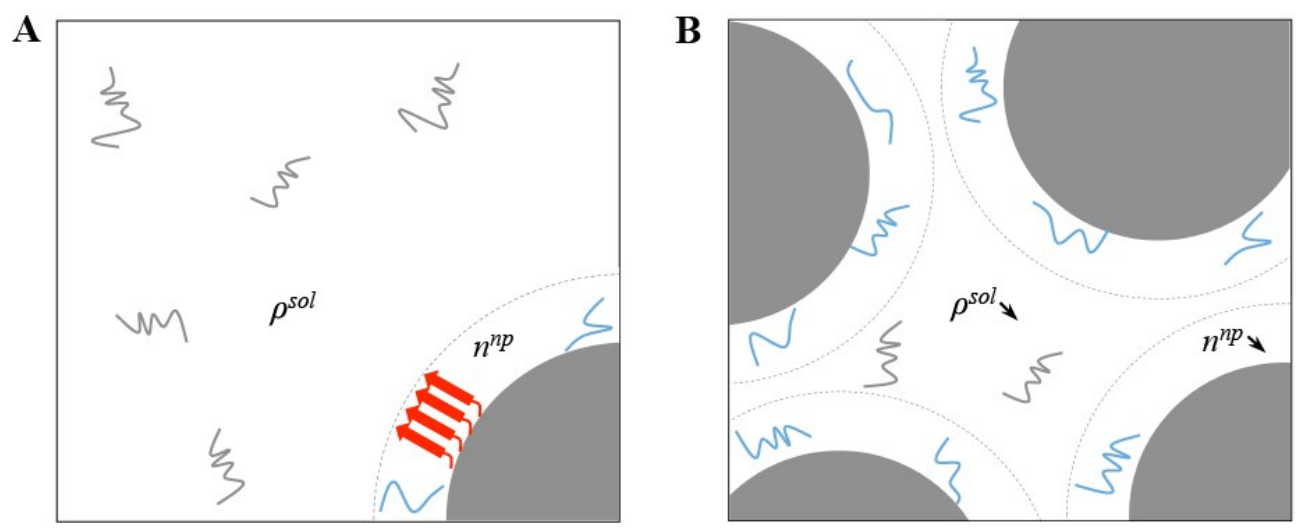

Figure 4. The effect of relative NP/peptide concentration on peptide aggregation. (A) With a high peptide to NP (depicted as gray solid spheres) ratio, the peptides in solution (denoted as curly lines in gray) are adsorbed onto the NP surface (denoted as lines in cyan). With a high local concentration of peptides on the NP surface that intrinsically promotes aggregation (relative weak NP-peptide attraction as in Fig. 2B), the peptides can self-associate and form amyloid fibrils (red arrows). (B) With reduced peptide to NP ratio due to increased NP concentration, the peptides in solution $\left(\rho^{s o l}\right)$ are depleted by binding NPs. Concomitantly, the concentration of proteins on NP surface $\left(n^{n p}\right)$ is also reduced compared to the case in panel (A). The decrease of peptide concentrations in solution and also on the NP surface leads to reduced amyloid aggregation. 


\title{
Contrasting effects of nanoparticle-protein attraction on amyloid aggregation
}

\author{
Slaven Radic, ${ }^{1}$ Thomas P Davis, ${ }^{2,3}$ Pu Chun Ke ${ }^{2}$ and Feng Ding ${ }^{1, *}$ \\ ${ }^{1}$ Department of Physics and Astronomy, Clemson University, Clemson, SC 29634, USA \\ ${ }^{2}$ ARC Centre of Excellence in Convergent Bio-Nano Science and Technology, Monash Institute of \\ Pharmaceutical Sciences, Monash University, 381 Royal Parade, Parkville, VIC 3052, Australia \\ ${ }^{3}$ Department of Chemistry, Warwick University, Gibbet Hill, Coventry, CV4 7AL, United Kingdom
}

*Correspondence to Feng Ding: fding@clemson.edu

\section{Supplementary Figures}
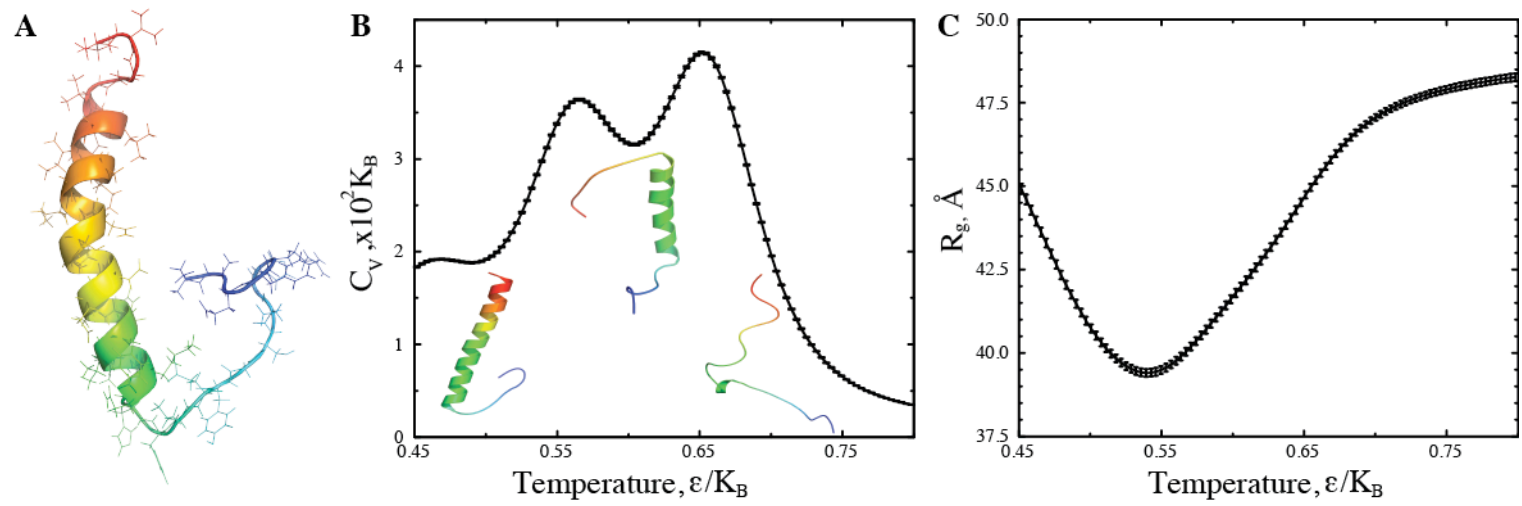

Figure S1. Folding thermodynamics of $A \beta$ monomer derived from DMD simulations. (A) The native state of $\mathrm{A} \beta$ (PDB: 1BA4) is shown in cartoon representation. The backbone trace form $\mathrm{N}$ - to $\mathrm{C}$-terminal is colored in rainbow for blue to red, respectively. (B) Specific heat $\left(C_{v}\right)$ and (C) Radius of gyration $\left(R_{g}\right)$ and corresponding g statistical uncertainties (as error bars) were computed from replica exchange DMD simulations using the WHAM analysis. Typical structures of $A \beta$ in the simulations, corresponding to native-like, intermediate, and unfold states, are shown in the inset of panel B. 


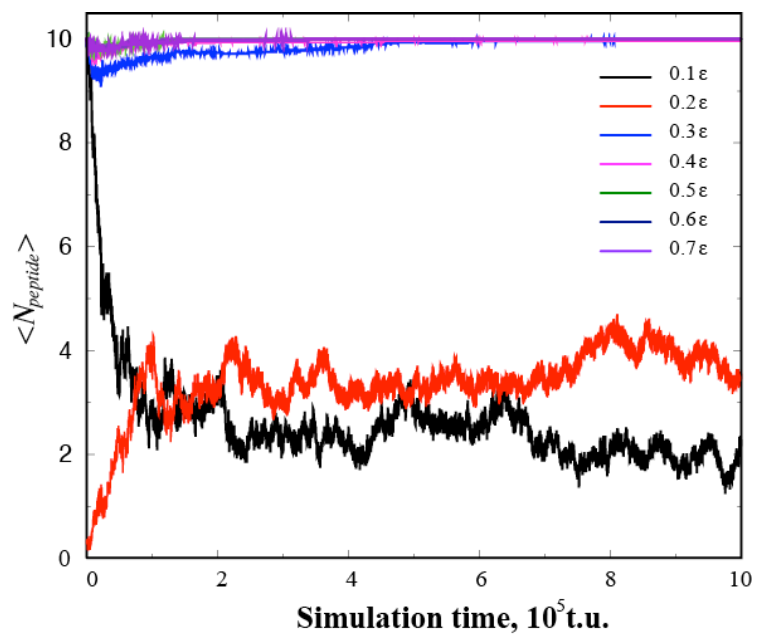

Figure S2. For different values of NP-protein interaction strength, the average number of A $\beta$ peptides on the NP surface was computed as a function of simulation time. The average was taken from 50 independent simulations, each of which was performed for a total $1 \times 10^{6}$ time units (t.u.). 

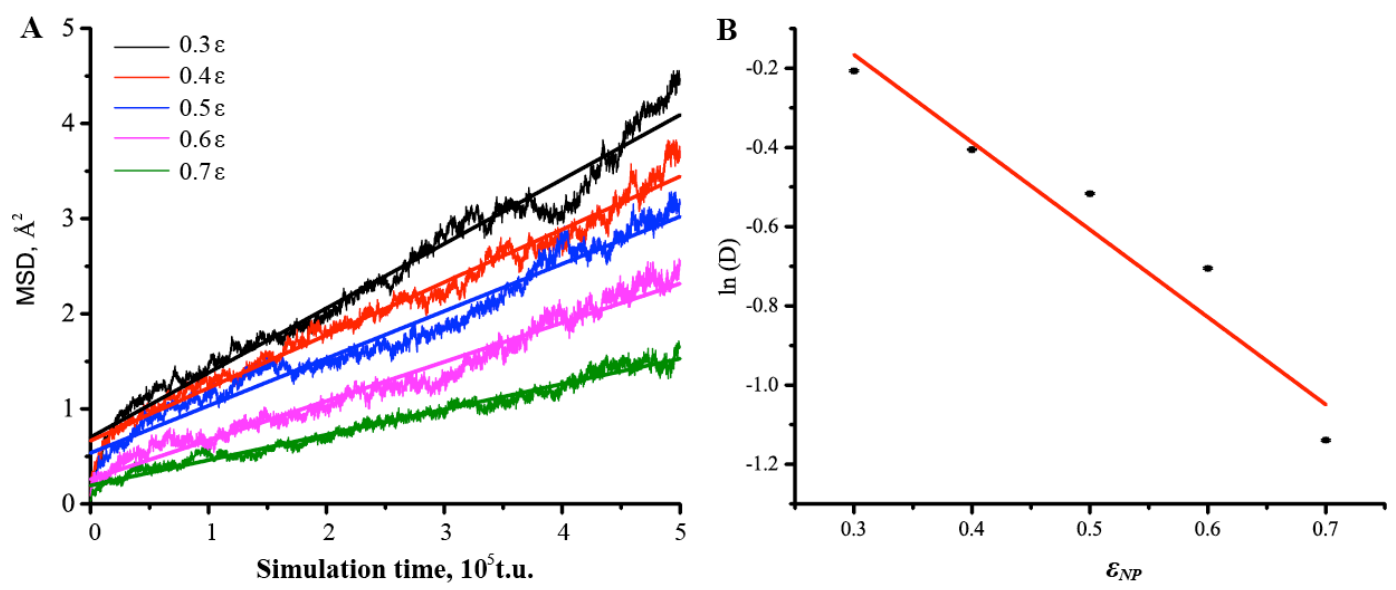

Figure S3. Peptide diffusion on the NP surface. (A) Mean square deviation (MSD) of the peptides was computed as a function of its diffusion time on the NP surface. The analysis was done for simulations with a single peptide bound to the NP surface. We only performed simulations with $\varepsilon_{N P} \geq 0.3 \varepsilon$ such that the peptide stayed as bound (e.g. Fig. S1). Linear-fit results in the diffusion coefficients, $D$. (B) The diffusion coefficients follow a linear dependence on NPprotein interaction potential $\boldsymbol{\varepsilon}_{N P}$ in the log-linear plot, suggesting an exponential-like dependence $D \sim \exp \left(-c \boldsymbol{\varepsilon}_{N P}\right)$. 


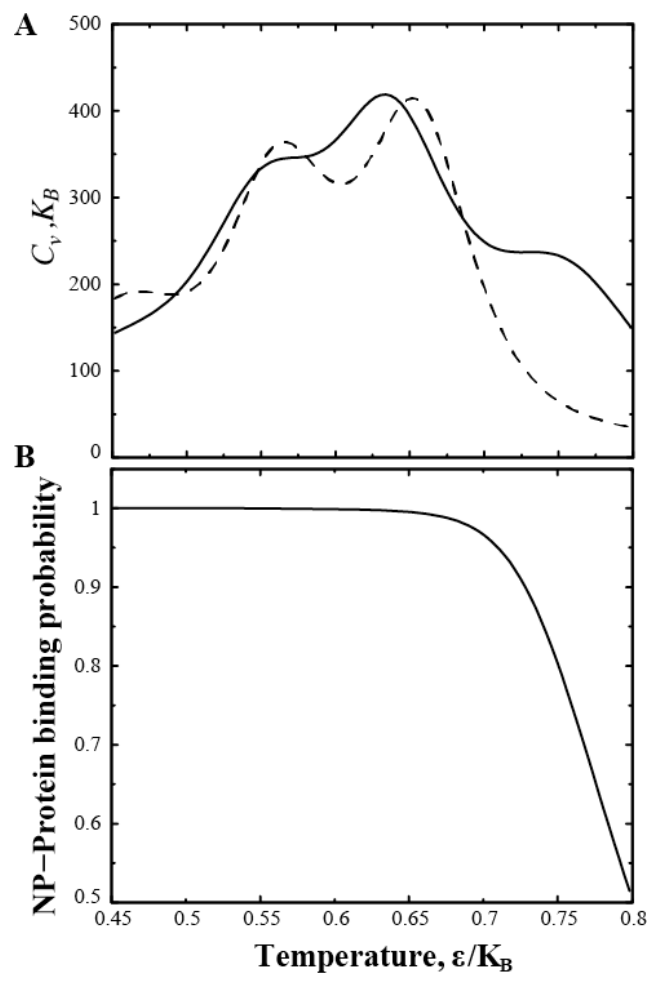

Figure S4. (A) Heat capacity of $\mathrm{A} \beta$ monomer in the presence of the NP (solid line), compared to the corresponding heat capacity in the absence of the NP (dashed line). (B) The binding probability of the peptide to the NP surface. 

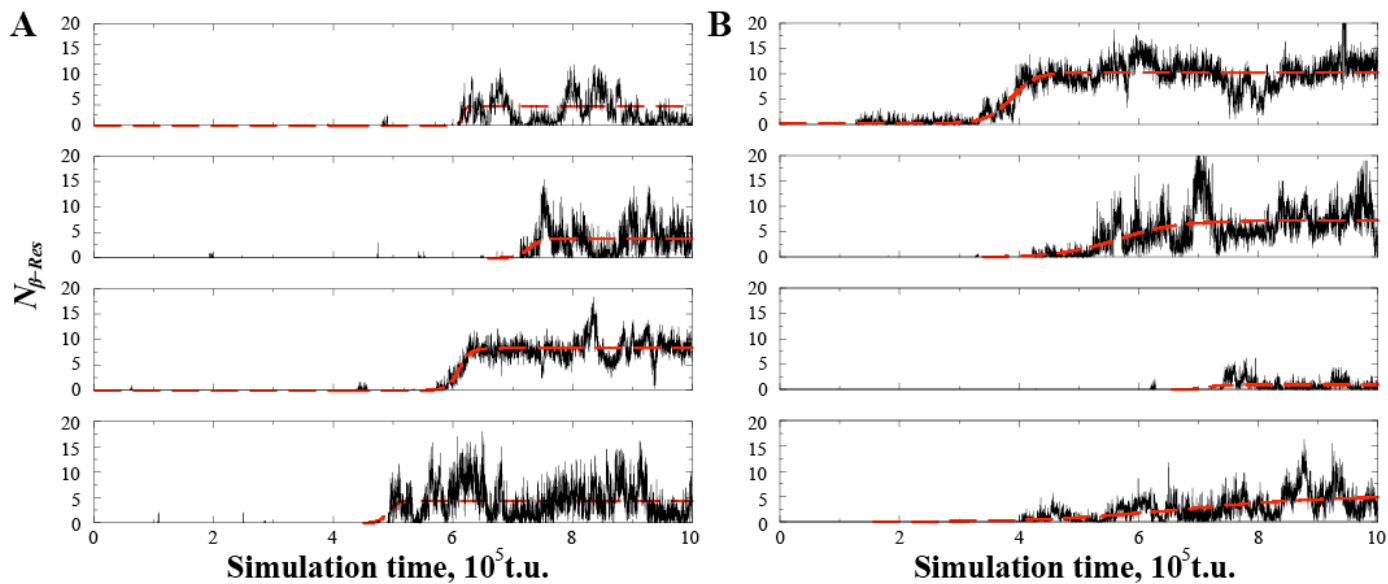

Figure S5. Typical trajectories (4 out of 50 independent simulations) of $N_{\beta \text {-res }}$ for proteins in solution (not NP-bound) as a function of simulation time, in the absence (A) and presence (B) of NP-protein attraction. For each trajectory, the sigmoidal fit was shown as a red dashed line. 


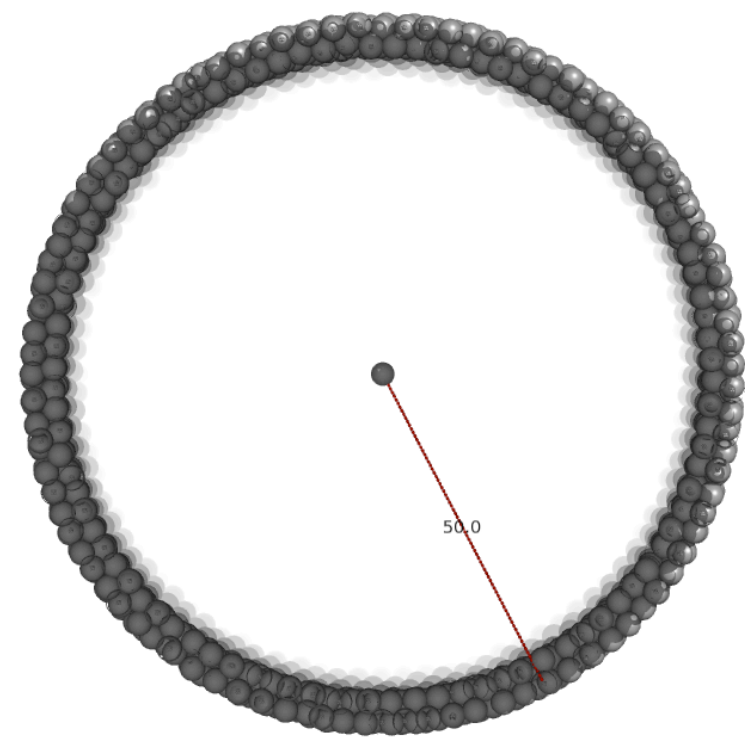

Figure S6. The cross-section of the NP model indicates the two layers of closely packed surface atoms. 